\title{
UM MÉTODO PARA AUXILIAR PROCESSOS DE RESTAURAÇÃO DE SISTEMAS DE POTÊNCIA
}

\author{
José V. Canto dos Santos* \\ jvcantodeuler.unisinos.br \\ Ariovaldo V. Garcia ${ }^{\dagger}$ \\ ari@dsee. fee.unicamp.br \\ ${ }^{*}$ Centro de Ciências Exatas e Tecnológicas, UNISINOS CP 275, 93022-000 São Leopoldo - RS, Brasil. \\ ${ }^{\dagger}$ DSEE, FEEC, UNICAMP CP 6101, 13081-970 Campinas - SP, Brasil.
}

\begin{abstract}
The subject of this work is the power system restoration, one of the main problems in the electrical engineering area. Due to the great dependency of electricity of a modern industrial society, this subject has receiving great attention. The restoration of large electrical power systems after the occurrence of serious blackouts is a complex problem where the basic goal is to obtain the system configuration in order to attend loads with different priorities. The restoration is done through stages and in each stage the service is restored to a predetermined set of loads. A method to solve important aspects of the power system restoration problem is presented in this work. The solution takes into account the nonlinear network model (AC model) as well as its constraints and operational limits. The fictitious network concept is extended to the reactive model, linear programming, a new model for the linearized power flow and conventional load flow calculation are used. Results obtained with a test system as well as with a large realistic Brazilian system are presented.
\end{abstract}

KEYWORDS: Power System Restoration, linearized power flow, security analysis.

\section{RESUMO}

Este trabalho trata de restauração de sistemas de potência, um dos principais problemas atuais da área de Engenharia

\footnotetext{
Artigo submetido em 12/12/02

1a. Revisão em 28/04/03

Aceito sob recomendação do Ed. Assoc. Prof. Glauco N. Taranto
}

Elétrica. Devido a grande dependência das sociedades industriais modernas de um fornecimento confiável de energia elétrica, este tema tem recebido grande atenção. Na restauração de sistemas após a ocorrência de blecautes, um problema básico é a determinação da configuração do sistema tendo como objetivo o atendimento de cargas prioritárias. Isso normalmente é feito em estágios sendo que em cada etapa atendese um conjunto de cargas. Neste trabalho é apresentado um método para resolver este problema levando em conta os aspectos ativo e reativo dos sistemas e seus principais limites de operação. A abordagem de caráter geral desenvolvida utiliza, um novo modelo de fluxo de potência linearizado, programação linear, uma expansão do conceito de rede fictícia. e o modelo convencional de fluxo de potência. São apresentados resultados obtidos para um sistema teste e para um sistema real brasileiro.

PALAVRAS-CHAVE: Restauração de sistemas de potência, fluxo de potência linearizado, análise de segurança.

\section{INTRODUÇÃO}

A ocorrência de blecautes envolvendo grandes seções dos sistemas de energia elétrica ainda é uma possibilidade real, mesmo após a realização de grandes investimentos para a melhoria de sua segurança. Os prejuízos causados à sociedade por esses blecautes são imensos. Com o continuo crescimento da complexidade dos sistemas e da demanda de energia elétrica é necessário que o tratamento do sistema após a ocorrência de blecautes faça parte dos procedimentos de operação. É nesse contexto que a restauração de sistemas 
de potência (RSP) tem recebido especial atenção nos últimos anos, sendo que na literatura sobre o assunto percebe-se que a solução geral do problema ainda não foi encontrada.

Os trabalhos que utilizam técnicas de Inteligência Artificial para tratar da restauração são numerosos, destacando-se o desenvolvimento de sistemas especialistas para o apoio à tomada de decisão dos operadores dos sistemas quando a restauração se faz necessária. Alguns exemplos são os artigos de Sakaguchi, e Matsumoto (1983), Kirschen e Volkman (1991), Matsumoto et al. (1992) e Park e Lee (1997). É interessante notar que, acredita-se, o trabalho de Sakaguchi, e Matsumoto (1983) corresponde à primeira proposta de construção de um sistema especialista para o problema de RSP e, também à uma das primeiras aplicações de técnicas de Inteligência Artificial para a área de análise de sistemas de energia elétrica. Nesse tipo de abordagem, um ponto fundamental é a utilização da experiência que os operadores têm sobre os sistemas na construção de regras de conhecimento utilizadas em sistemas especialistas. É de se esperar que exista um certo grau de dependência entre o sistema elétrico considerado e o sistema especialista desenvolvido.

Outra abordagem para o tema baseia-se no uso de técnicas de otimização, presente em um numero menor de trabalhos, destacando-se os de Wu e Monticelli (1988) e de Huang et al. (1995). Segundo Wu e Monticelli (1988) o problema é não linear com restrições, combinatorial, multiestágio e multiobjetivo. Neste trabalho são sugeridos diferentes tipos de programação matemática para os vários aspectos da RSP. Salienta-se que, considerando que o procedimento de restauração será determinado após o blecaute, uma restrição fundamental é o período no qual o sistema fica sem fornecimento de energia, ou seja, a formulação adotada deve permitir a solução rápida do problema. Esta observação é válida mesmo quando se consideram os recursos computacionais de maior desempenho atualmente existentes.

Existem outras publicações que enfocam particularidades importantes do problema, como pode ser visto, por exemplo, em Adibi et al. (1992). Segundo os autores, usos não convencionais e relativamente imprevisíveis dos sistemas de energia elétrica estão sendo feitos em um período em que os sistemas operam desconfortavelmente próximos de seus limites operativos. Isto torna as redes mais vulneráveis à possibilidade de grandes distúrbios, o que resulta em uma renovada atenção para a necessidade de restauração pronta e efetiva após a ocorrência de blecautes. Alguns problemas enfocados são: o número excessivo de alarmes nos centros de controle, as operações de chaveamento, o seqüenciamento ótimo de partida de unidades geradoras e os aspectos relativos a sistemas de transmissão subterrâneos.

Neste trabalho é apresentado um método que resolve um dos problemas básicos em procedimentos de restauração: a determinação, em cada estágio do processo, de configurações do sistema adequadas para o atendimento de cargas prioritárias. O método considera, no aspecto estático, as partes ativa e reativa dos sistemas e seus principais limites de operação. As soluções obtidas podem ser utilizadas para a determinação de procedimentos de restauração após a ocorrência de blecautes bem como em métodos de planejamento de tais procedimentos. A proposta apresentada neste artigo é parte integrante da metodologia para recomposição de redes elétricas de grande porte apresentada no trabalho de Canto dos Santos e Garcia, 1998a.

O aspecto dinâmico do problema é um dos fatores que eleva consideravelmente o seu grau de complexidade, devido principalmente à dificuldade de incorporação dos modelos matemáticos necessários em métodos de solução. Durante a recomposição de um sistema são feitas inúmeras manobras de chaveamento de equipamentos, fazendo que a topologia da rede mude constantemente até que seja obtida uma configuração capaz de resolver um dos estágios do problema. Após a determinação desta configuração, deve-se verificar se a mesma é satisfatória quanto à estabilidade. Isto deve ser feito para cada estágio do processo. Além desse fato, normalmente a recomposição de sistemas de grande porte é feita através da reenergização de partes (ilhas) independentes dos sistemas. Apenas no final do processo são efetuadas as manobras para a interligação das várias ilhas. Assim, um ponto importante no aspecto dinâmico do problema é a manutenção do sincronismo entre geradores no final da recomposição da rede. Existem outras particularidades do problema de restauração que se relacionam com os diferentes aspectos do problema de estabilidade, conforme pode ser visto sinteticamente em Canto dos Santos e Garcia, 1998a. Cabe lembrar que mesmo quando se trata apenas do aspecto estático do problema, este já é bastante complexo. A consideração do aspecto dinâmico está sendo analisada e será tema de outro artigo.

\section{FORMULAÇÃO DO PROBLEMA}

Restaurar um sistema de potência significa determinar a melhor forma de conduzir o sistema de um estado onde, devido a contingências graves, sua integridade está prejudicada, a outro onde cargas consideradas prioritárias sejam atendidas e limites de operação respeitados. O problema é do tipo multiestágio, sendo que em cada estágio o objetivo é o restabelecimento do serviço a um grupo de cargas prioritárias e a restrição mais importante é o período em que consumidores ficam sem energia. O processo de restauração é complexo, mesmo em seu aspecto estático, devido ao grande número de fatores envolvidos. Entre esses fatores pode-se realçar a identificação e escalonamento dos recursos de geração dispo- 
níveis, os equipamentos disponíveis para serem reconectados (linhas, transformadores, bancos shunt) e os limites operativos de todos os equipamentos utilizados ou a serem reconectados (limites para injeções de potência ativa e reativa em geradores e limites nas capacidades de transmissão das linhas e transformadores).

Neste trabalho, para a solução do problema proposto, partese do ponto em que o sistema de potência (ou parte dele) esteja fora de operação; isto é, eventos que levaram o sistema para o estado restaurativo não são analisados. Em cada estágio do processo, são considerados conhecidos as cargas prioritárias e os equipamentos da rede que estão em condições de serem religados para a recomposição do sistema.

\subsection{Tratamento de sistemas desconexos.}

Em um blecaute é freqüente a separação do sistema em diversos subsistemas (ilhas) devido a perda de interligações. Ainda, uma das recomendações que aparecem constantemente nos procedimentos para se restaurar o sistema consiste exatamente em abrir todos os disjuntores para permitir a reconstrução da rede (Adibi et al., 1992). Neste trabalho, para se trabalhar com sistemas desconexos, utiliza-se uma rede fictícia, procedimento proposto anteriormente para o planejamento da expansão do sistema de transmissão - Monticelli et al. (1982). Cada ramo do sistema que está fora de operação é substituído por um ramo fictício com impedância artificialmente elevada. Isso possibilita que a rede analisada seja sempre conexa (portanto não havendo matrizes singulares nas soluções do tipo $\mathrm{A} x=b$ ) além de permitir a verificação de caminhos por onde há necessidade de fluxo de potência. Neste trabalho o conceito de rede fictícia é estendido para a representação de resistências, reatâncias e admitâncias shunt de ramos e admitâncias shunt de barras.

\subsection{Modelo alternativo de fluxo de potên- cia linearizado.}

Na RSP, um problema a ser resolvido é, uma vez conhecidas as cargas prioritárias, descobrir qual ou quais geradores deverão, atender essas cargas. Esse problema é mais crítico no início do processo, pois podemos ter diversos geradores para atender poucas cargas. Para evitar um problema de otimização (despacho de geração) nesta etapa, foi desenvolvido um novo modelo de fluxo de potência linearizado (Canto dos Santos e Garcia, 1998b) que de uma maneira muito simples e eficiente, determina automaticamente os geradores "mais próximos" das cargas e, ainda, fornece a potência solicitada a cada gerador. Como será visto adiante, possíveis violações de limites operativos são tratadas em seguida.

Considere-se o modelo linearizado "convencional" de fluxo

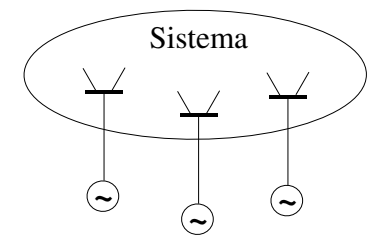

a)

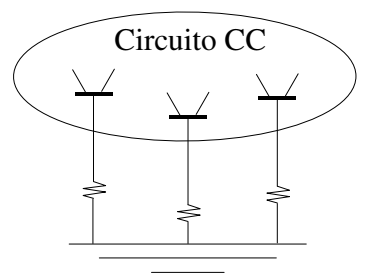

b)
Figura 1: Modelo alternativo de fluxo de potência linearizado: a) Sistema CA; b) Modelo CC com barras terminais dos geradores ligadas à terra.

de potência (FCCC). Nesse modelo, o sistema de potência é tratado como um circuito de corrente contínua onde as reatâncias são tratadas como resistências, fluxos e injeções de potência ativa são tratados como fluxos e injeções de corrente e, finalmente, os ângulos de tensões das barras são tratados como tensões contínuas. Usando esse modelo o fluxo de potência se resume na solução do sistema linear:

$$
P=B^{\prime} \theta
$$

com os elementos da matriz $B /$ dados por

$$
\begin{gathered}
B_{k m}^{\prime}=-x_{k m}^{-1} \\
B_{k k}^{\prime}=\sum_{m \in \Omega k} x_{k m}^{-1}
\end{gathered}
$$

onde $\Omega_{k}$ é o conjunto das barras vizinhas da barra $k, x_{k m}$ é a reatância do ramo cujos terminais são $k$ e $m, P$ é o vetor de injeções de potência ativa e $\theta$ é o vetor de ângulos. Dados $P$ e $B^{\prime}$ obtém-se $\theta$. No modelo original uma das barras do sistema é escolhida como barra de referência, tendo portanto seu ângulo especificado, para o sistema ter solução e todas as demais barras têm potência ativa especificada. No modelo usado neste trabalho adota-se o nó "terra" (circuito de corrente contínua) como referência e com tensão nula. A potência fornecida pelas barras com geradores é desconhecida antes da solução do sistema linear. Essas barras são ligadas à terra pela sua reatância (resistência no modelo CC) síncrona, conforme esquematizado na Figura 1. É possível verificar que se as reatâncias síncronas em p.u. tiverem valores próximos, então uma boa aproximação para esses valores é usar os inversos das potências nominais máximas de cada gerador, em $p . u$. Sendo $S_{b}$ a potência de base do sistema, $S_{g e r}$ e $V_{g e r}$ a potência e a tensão nominais de um determinado gerador, $x_{\text {orig }}$ sua reatância em p.u. na base $\left(S_{g e r}, V_{g e r}\right)$, a reatância em p.u. na base do sistema será (assumindo que a tensão de base do sistema seja igual à do gerador):

$$
x_{p . u .}=x_{\text {orig }} \times \frac{V_{\text {ger }}^{2}}{S_{\text {ger }}} \frac{S_{b}}{V_{\text {ger }}^{2}}
$$


Logo

$$
x_{p . u .}=x_{\text {orig }} \times \frac{S_{b}}{S_{g e r}}=\frac{x_{\text {orig }}}{S_{g e r} / S_{b}}
$$

Se todos os geradores tiverem o mesmo $x_{\text {orig }}$, a relação entre as reatâncias em p.u.será igual à relação inversa das potências nominais dos geradores expressas em p.u. na base do sistema. No nosso caso, utilizamos para cada gerador $i$ o inverso da potência ativa máxima permitida para o gerador como sendo o valor da reatância:

$$
x g_{i}=\frac{1}{P g_{i}^{\max }}
$$

Deve-se notar que, neste modelo, as barras terminais dos geradores têm potência especificada nula. A potência que o gerador irá fornecer será calculada após a solução do sistema $P=B ı$. A potência fornecida pelo gerador $i$ será:

$$
P g_{i}=\frac{-\theta_{i}}{x g_{i}}
$$

onde $P g_{i}$ é a potência ativa gerada na barra $i$ e $\theta_{i}$ é o ângulo da barra $i$. Logo, o modelo alternativo aqui proposto é de certa forma, um modelo com referência distribuida. Se o sistema elétrico for forte o suficiente, mostra-se que a distribuição da geração entre os geradores se dá na razão de sua potência máxima. Esse último fato é fácil de verificar considerando a idéia de divisor de correntes - modelo CC.

Finalmente, o novo modelo linearizado em conjunto com a utilização de ligações fictícias permite que se trabalhe com sistemas desconexos e, ainda, através da verificação dos fluxos de potência (correntes) nas ligações fictícias pode-se decidir sobre a necessidade de se religar ou não um dado ramo, conforme visto mais adiante.

\section{ABORDAGEM PROPOSTA}

A solução para cada estágio do problema é obtida através de duas fases principais. Assume-se que em cada estágio foram definidos os equipamentos (linhas, transformadores, geradores, reatores, etc.) disponíveis para serem colocados em operação e as cargas a serem atendidas. Normalmente o total de cargas prioritárias por estágio é crescente e no último estágio deseja-se a restauração do sistema inteiro. Cada fase é descrita de maneira resumida a seguir.

Fase I - Problema “CC”.

Determina quais ramos têm que ser religados para atender a demanda "ativa". Esta Fase é composta de 2 etapas.

I.1. Determinar ramos a serem religados utilizando a rede fictícia (considera-se que existe uma linha fictícia onde existir uma linha ou transformador que possa ser religado) e o modelo alternativo de fluxo de potência linearizado descrito na seção anterior. Sucessivamente resolve-se um FCCC alternativo e aloca-se o ramo fictício mais carregado (quando um ramo é religado os parâmetros fictícios são substituídos pelos reais) até que não exista fluxo considerável passando pela rede fictícia - ver Figura 2.

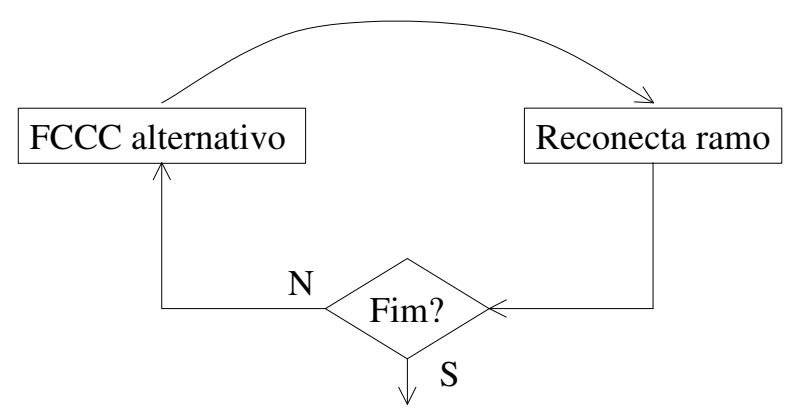

Figura 2: Visão simplificada da etapa I.1

I.2. Terminada a etapa I.1, caso existam violações ou nas injeções dos geradores ou em fluxos nos ramos em operação, realiza-se um despacho de potência ativa via programação linear (PL) e, caso necessário, novos ramos são religados. Visando o atendimento integral de cargas consideradas prioritárias, o corte de carga não é permitido nesta fase. Por este motivo, o cálculo de PL pode ser infactível. Nesse caso, sucessivamente, aloca-se o ramo mais carregado na última solução obtida via modelo alternativo de fluxo de potência linearizado e executa-se nova PL. Quando a PL apresenta solução, o modelo linearizado de fluxo de potência é o convencional (com uma das barras escolhida como referência). A PL é quem determina o quanto gerar em cada gerador de modo que nestes os limites serão respeitados. Visando manter a solução próxima da obtida na etapa I.1, no problema de PL procura-se uma solução em que os limites são respeitados porém com mínimo desvio do ponto de operação determinado nesta etapa. O problema resolvido é do tipo descrito nas equações a seguir.

$$
M i n C^{\prime} \Delta P_{I}+C^{\prime} \Delta P_{I I}
$$

sujeito a:

$$
\begin{gathered}
\sum_{i=1}^{n b} \Delta P_{I}-\sum_{i=1}^{n b} \Delta P_{I I}=0 \\
0 . \leq \Delta P_{I} \leq P^{\max }-P^{0} \\
0 . \leq \Delta P_{I I} \leq P^{0} \\
P_{k m}^{\min }-P_{k m}^{0} \leq \Delta P_{k m} \leq P_{k m}^{\max }-P_{k m}^{0}
\end{gathered}
$$

onde: 
nb - número de barras do sistema,

$C$ - custos,

$\Delta P_{I}$, e $\Delta P_{I I}$ - incrementos de aumento e de redução nas injeções $P^{0}$,

$P^{0}$, e $P^{\max }$ - potências ativas iniciais e limites máximos para injeções,

$P_{k m}^{0}, \quad P_{k m}^{\min }, P_{k m}^{\max }$ e $\Delta P_{k m}$ - fluxos de potência ativa na solução inicial, limites e incrementos para fluxos. Ao final desta fase, temos uma rede composta por ramos, geradores e cargas com todos os limites atendidos.

\section{Fase II - Problema “CA”}

Nesta fase a rede obtida na Fase I será testada e, se necessário, alterada para considerar a parte reativa do sistema (limites de tensão, fontes reativas, etc.). Equipamentos ainda não religados continuam sendo representados por parâmetros fictícios. Esta fase é composta de três etapas, conforme será visto a seguir.

II.1. Realiza-se um despacho reativo (uma referência sobre o tema é o trabalho de Mantovani e Garcia, 1996) para a rede tendo como função objetivo o custo associado a utilização de fontes reativas. Neste despacho, os limites para tensão são relaxados em barras ainda não religadas e que possuem fontes não estáticas (geradores por exemplo) de potência reativa, permitindo ao algoritmo alocar potência reativa na barra se necessário. Já os limites para injeções reativas nas barras prioritárias são tais que o atendimento destas injeções é garantido. O modelo "CRIC" (Carpentier, 1986) é empregado na sensibilidade entre potência reativa e magnitudes de tensão, permitindo que se trabalhe com a potência reativa de forma praticamente independente da parte ativa.

II.2 Após a etapa II.1 verifica-se a necessidade do religamento de fontes reativas existentes em barras pertencentes à rede configurada na Fase I. Em barras dotadas de capacitores em que a tensão atingiu o limite mínimo no despacho reativo, os capacitores são religados. Analogamente, são religados os reatores presentes em barras em que a tensão atingiu o limite máximo. Posteriormente, verifica-se se a rede construída está em condições de operar. Isso é feito com um fluxo de potência não linear (FCAC) usando os dados obtidos nas etapas anteriores. Como o sistema pode estar operando separado em ilhas, deve-se tomar o cuidado de definir barras de referência para cada ilha. Se a solução for obtida, fim do estágio e início da solução do próximo. Caso contrário, deve-se realizar a fase II.3.

II.3 Nesta etapa verifica-se a necessidade de utilização de fontes reativas situadas em barras que não foram religadas na Fase I. Para dispositivos estáticos a verificação é a mesma da etapa anterior. Entre as outras fontes são consideradas necessárias aquelas que tiveram uma injeção reativa não desprezível na etapa II.1. As barras com fontes reativas consideradas necessárias são religadas ao sistema com a respectiva fonte (síncrono, reator, etc.). Nesse processo, a barra é incorporada ao sistema através do religamento dos ramos com maior fluxo reativo até que uma barra já "energizada" seja alcançada. Posteriormente, é calculado o fluxo de potência para a nova rede configurada, tal como na etapa II.2. Se este cálculo tem solução, chegou-se fim do estágio e dá-se início a solução do próximo. Caso contrário deve-se realizar novamente a etapa II. 1 com novos dados.

\section{TESTES}

Na implementação feita utilizou-se PL dual e o software MINOS. Os módulos foram escritos em Fortran e os testes efetuados em estações Sun Ultra 1. Utilizou-se o fluxo de potência desacoplado rápido (Monticelli et al., 1990), versão BX, para o caso não linear.

\section{Sistema Teste IEEE-14.}

O sistema teste IEEE-14 (Freris e Sasson, 1968), por ser de pequeno porte é adequado para a descrição dos resultados obtidos. Para este sistema, considerou-se a ocorrência de um blecaute geral e que os geradores das barras 1 e 2 e todos os 20 ramos (linhas e transformadores) estavam fora de operação, porém disponíveis para a restauração da rede. Foram definidos dois estágios, sendo que no primeiro as cargas prioritárias foram as das barras 4 e 12 . O objetivo do segundo estágio é atender toda a carga da rede.

Na solução do primeiro estágio obteve-se inicialmente uma rede com 5 barras e 4 ramos. Em seguida a rede é expandida para 13 barras e 15 ramos no segundo estágio. Em ambos os estágios, o objetivo de atender às cargas prioritárias respeitando os limites existentes foi atingido, sendo que a faixa permitida para módulos de tensão foi de 0.9 a 1.1 p.u. Na solução do primeiro estágio, as etapas I.2 e II.3 não precisaram ser realizadas. Este fato ocorreu em diversos testes com diferentes sistemas. Nas Tabelas 1 e 2 são fornecidos detalhes da solução e a topologia da rede configurada é mostrada na Figura 3.

\subsection{Sistema Real Brasileiro SB-810.}

Neste teste utilizamos uma configuração do sistema tema das regiões Sul e Sudeste do Brasil que possui 810 barras (82 geradores) e 1340 ramos. O teste aqui apresentado considerou a ocorrência de um blecaute em todo o sistema e que todos os seus componentes estavam disponíveis para serem reconectados a operação. Foram considerados dois estágios de restauração. No primeiro estágio o objetivo foi atender a 


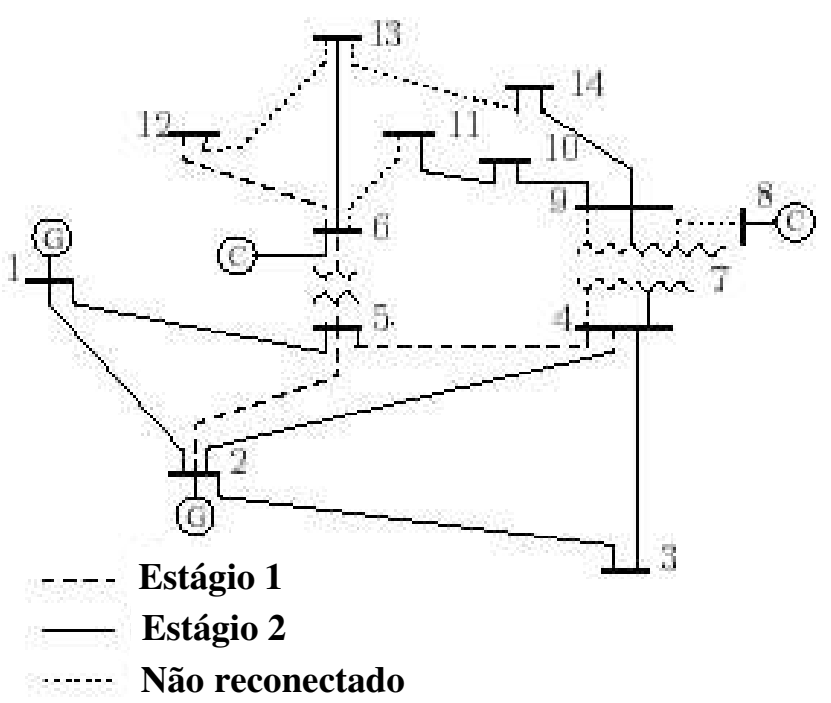

Figura 3: Solução obtida - sistema teste IEEE-14

Tabela 1: Testes com sistema IEEE-14. Solução do primeiro estágio

\begin{tabular}{|l|c|}
\hline Barras religadas na etapa I.1 & 245612 \\
\hline Ramos religados na etapa I.1 & $2-5,4-5,5-6,6-12$ \\
\hline Equipamentos religados - F. II & - \\
\hline Cálculos de FCAC na etapa II.2 & 1 \\
\hline $\mathrm{N}^{o}$ de geradores usados & 1 \\
\hline $\mathrm{N}^{o}$ de ramos em atividade & 4 \\
\hline
\end{tabular}

Tabela 2: Testes com sistema IEEE-14. Solução do segundo estágio

\begin{tabular}{|l|c|}
\hline \multicolumn{2}{|c|}{ Solução do segundo estágio } \\
\hline Barras religadas na etapa I.1 & 37910111314 \\
\hline Ramos religados na etapa I.1 & $3-4,4-7,7-9,6-13$ \\
& $9-10,9-14,10-11$ \\
\hline Barras religadas na etapa I.2 & 1 \\
\hline Ramos religados na etapa I.2 & $1-2,2-3,1-5,2-4$ \\
\hline Equipamentos religados - F. II & - \\
\hline Cálculos de FCAC na etapa II.2 & 1 \\
\hline $\mathrm{N}^{o}$ de geradores usados & 2 \\
\hline $\mathrm{N}^{o}$ de ramos em atividade & 15 \\
\hline
\end{tabular}

apenas 2 cargas importantes (parte das cargas das cidades de Belo Horizonte e São Paulo) totalizando 1027 MW. No segundo e último estágio o objetivo foi o atendimento de todas as cargas do sistema, o que corresponde a aproximadamente $40000 \mathrm{MW}$. O perfil de tensão requerido neste teste foi uma faixa de 0.8 a 1.2 p.u.

Na solução do primeiro estágio, a configuração de sistema obtida consistiu de 2 sub-redes (designadas como A e B) em

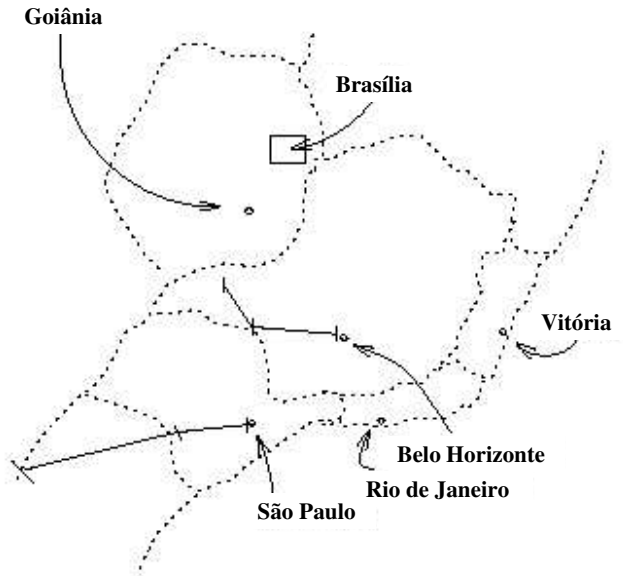

Figura 4: Solução obtida - parte do sistema sudeste.

Tabela 3: Teste com sistema SB-810. Solução do primeiro estágio $\mathrm{N}^{o}$ de barras religadas - et. I.1 13 $\mathrm{N}^{o}$ de ramos religados - et. I.1 11 Equipamentos religados - F. II 1 Cálculos de FCAC na etapa II.2 1 $\mathrm{N}^{o}$ de geradores em atividade 4

\begin{tabular}{|l|l|}
\hline $\mathrm{N}^{o}$ de barras em atividade & 13 \\
\hline $\mathrm{N}^{o}$ de ramos & 11 \\
\hline
\end{tabular}
\begin{tabular}{|l|l|}
$\mathrm{N}^{o}$ de ramos em atividade & 11 \\
\hline
\end{tabular}

Tabela 4: Teste com sistema SB-810. Primeiro estágio - subrede "A"

\begin{tabular}{|l|c|}
\hline \multicolumn{2}{|c|}{ Primeiro estágio - subrede "A" } \\
\hline $\mathrm{N}^{o}$ de barras & 3 \\
\hline $\mathrm{N}^{o}$ de ramos & 2 \\
\hline $\mathrm{N}^{o}$ de geradores em atividade & 1 \\
\hline Potência ativa prioritária & $526,00 \mathrm{MW}$ \\
\hline
\end{tabular}

Tabela 5: Teste com sistema SB-810. Primeiro estágio - subrede "B"

\begin{tabular}{|l|c|}
\hline $\mathrm{N}^{o}$ de barras & 10 \\
\hline $\mathrm{N}^{o}$ de ramos & 9 \\
\hline $\mathrm{N}^{o}$ de geradores em atividade & 3 \\
\hline Potência ativa prioritária & $501,00 \mathrm{MW}$ \\
\hline
\end{tabular}

operação independente e, a exemplo do teste com o sistema IEEE-14, foi obtida sem necessidade de realização das etapas I.2 e II.3. As Tabelas 3 a 5 mostram a solução obtida para o primeiro estágio e a Tabela 6 a solução do segundo estágio simulado. Na Figura 4 é representada a rede obtida no primeiro estágio 
Tabela 6: Teste com sistema SB-810. Solução do segundo estágio

\begin{tabular}{|l|c|}
\hline $\mathrm{N}^{o}$ de barras religadas - et. I.1 & 747 \\
\hline $\mathrm{N}^{o}$ de ramos religados - et. I.1 & 1198 \\
\hline $\mathrm{N}^{o}$ de barras religadas - et. I.2 & 0 \\
\hline $\mathrm{N}^{o}$ de ramos religados - et. I.2 & 14 \\
\hline Equipamentos relig. - et. II.2 & 6 \\
\hline Cálculos de FCAC - et. II.2 & 1 \\
\hline $\mathrm{N}^{o}$ de geradores em atividade & 82 \\
\hline $\mathrm{N}^{o}$ de barras em atividade & 760 \\
\hline $\mathrm{N}^{o}$ de ramos em atividade & 1223 \\
\hline
\end{tabular}

\section{CONCLUSÃo}

Neste trabalho foi apresentado um método para auxiliar processos de restauração de sistemas de potência, resolvendo um dos problemas básicos em tais procedimentos: a determinação de qual a configuração do sistema adequada para o atendimento de cargas prioritárias, em cada estágio do processo. Além de programação linear, o método utiliza um novo modelo de fluxo de potência linearizado e uma expansão do conceito de rede fictícia. Sendo que estes dois últimos desenvolvimentos podem ser aplicados em outras áreas da análise de sistemas de energia elétrica. A abordagem desenvolvida, simples porém robusta, traduz-se em um método analítico que pode ser aplicado a qualquer rede elétrica de potência cuja restauração após blecautes se faça necessária e que permite uma modelagem fácil de circunstâncias típicas de um sistema no estado restaurativo. Nas simulações realizadas os resultados obtidos foram plenamente satisfatórios e a eficiência computacional necessária para execução em tempo real foi atingida. Mostrou-se que, em cada estágio do processo de restauração, dependendo da definição do conjunto de barras de carga prioritárias, o número de equipamentos necessários para recompor o sistema pode ser consideravelmente menor do que o total de equipamentos da rede. Esse fato era esperado já que normalmente os sistemas operam com uma margem de segurança.

Foi apresentado um método que soluciona um dos subproblemas fundamentais do problema de restauração de sistemas de potência. A própria complexidade deste problema serve como um excelente indicativo para os desenvolvimentos futuros que podem ser acrescentados à metodologia desenvolvida. Assim, um prolongamento natural deste trabalho é a consideração de outros aspectos do problema, logicamente tendo como ponto de partida as configurações de sistemas obtidas com a presente metodologia. Alguns exemplos destas possibilidades são: a consideração do aspecto dinâmico do processo de recomposição de uma rede elétrica de grande porte através de cálculos de estabilidade (este é considerado o desenvolvimento mais necessário e está sendo pesquisado atualmente), a inclusão de mais variáveis na modelagem desenvolvida, idealmente com manutenção da eficiência computacional necessária para execução em tempo real, e a consideração de características dos geradores instalados em uma rede no que diz respeito á sua seqüência de partida e capacidade de assumir ou rejeitar carga. Outros desenvolvimentos futuros do presente trabalho considerados possíveis são a utilização de processamento paralelo ou distribuído na solução de problemas de restauração; a utilização de outras técnicas de análise já empregadas com sucesso na solução do problema de planejamento da expansão de sistemas de transmissão, como a Decomposição de Benders e os algoritmos de otimização combinatorial; e o desenvolvimento de um modelo não linear de cálculo de fluxo de potência que utilize a hipótese básica do modelo alternativo de FCCC, proposto neste trabalho.

\section{REFERÊNCIAS}

Adibi, M. et al. (1992). Special Considerations in Power System Restoration. IEEE Transactions on PWRS, EUA, 7(4): 1419-1427.

Canto dos Santos, J. V., Garcia, A. (1998a). Métodos Analíticos Para Auxílio à Restauração Após Blecautes de Sistemas de Energia Elétrica. Tese de Doutorado, Faculdade de Engenharia Elétrica - Universidade Estadual de Campinas, Brasil.

Canto dos Santos, J. V., Garcia, A. (1998b). Um Modelo Alternativo Para o Cálculo de Fluxo de Potência Linearizado. Anais do XII CBA, Brasil, p.1961-1966.

Carpentier, J. (1986). CRIC, a New Active Reactive Decupling Process in Load Flows, Optimal Power Flow and System Control. Proceedings IFAC Conference on Power Systems and Power Plan Control, China, p.6570.

Freris, L., Sasson, A. (1968). Investigation of the Load Flow Problem. Proceedings of IEE, Inglaterra, 115(10):1459-1470.

Huang, J. et al. (1995). A Systematic Method for Power System Restoration Planning. IEEE Transactions on PWRS, EUA, 10(2): 869-875.

Kirschen, D., Volkman, T. (1991). Guiding a Power System Restoration With an Expert System, IEEE Transactions on PWRS, EUA, 6(2): 558-566.

Mantovani, J., Garcia, A. (1996). A Heuristic Method for Reactive Power Planning. IEEE Transactions on PWRS, EUA, 11(1): 68-74. 
Matsumoto, K. et al. (1992). Knowledge-Based Systems as Operational Aids in Power Systems Restoration. Proceedings of the IEEE, EUA, 80(5): 689-697.

Monticelli, A. et al. (1982). Interactive Transmission Network Planning Using a Least-Effort Criterion. IEEE Transactions on PAS, EUA, 101(10): 3919-3925.

Monticelli, A. et al. (1990). Fast Decupled Load Flow: Hypothesis, Derivations and Testing. IEEE Transactions on PWRS, EUA, 5(4):1425-1431.

Park, Y., Lee, K. (1997). Application of Expert System to Power System Restoration in Sub- Control Center. IEEE Transactions on PWRS, EUA, 12(2): 629-635.

Sakaguchi, T.,Matsumoto, K. (1983). Development of a Knowledge Based System for Power System Restoration. IEEE Transactions on PAS, EUA, 102(2): 320-329.

Wu, F., Monticelli, A. (1988). Analytical Tools for Power System Restoration - Conceptual Design. IEEE Transactions on PWRS, EUA, 3(1):10-16. 